\title{
Optimal SAR Distribution from Waveguide Applicators for Hyperthermia of Deep-seated Tumours
}

\author{
R. W. Y. Habash and A. Kumar \\ E.C.E. Department, Indian Institute of Science, Bangalore-560 012, India.
}

\begin{abstract}
A major characteristic in the hyperthermia technology is the ability to focus heat energy on a small region around the tumour to be treated. By applying Kirchhoff-Huygens principle and the scalar theory of diffraction, specific absorption rate (SAR) distribution in a homogeneous muscle due to arrays of open-ended rectangular waveguide as directcontact applicators (ORWDAs) at $915 \mathrm{MHz}$ are calculated. The results are compared with that of a single ORWDA operating at the same frequency. The array applicators may offer improvement in field size and penetration depth.
\end{abstract}

\section{Introduction}

Although an ORWDA is commonly used in microwave hyperthermia, much attention has been drawn to arrays of ORWDAs [1-2]. These arrays have appeared to be the best approach to focus the energy, resulting in increased microwave penetration, and hence avoiding superficial skin burns and overheating the surrounding healthy tissues.

In this paper, we propose a simple configuration and method of determining the SAR distribution from the aperture of a linear and a planar array of ORWDAs, for forming a hot zone around deep-seated tumours. The main point of the method is to determine the near field patterns from the aperture of ORWDAs inside the human muscle using the Kirchhoff-Huygens principle and the scalar theory of diffraction. The performance of both cases of arrays is assumed in relation to the SAR distribution of a single ORWDA.

\section{Method}

The frequency to be considered is at the ISM band, $915 \mathrm{MHz}$. An ORWDA employing the rectangular metal waveguide, operating in its $T E_{10}$-mode, is selected. It is proposed that the interior of the waveguide be filled with the ceramic material magnesium calcium titanate. This material has a permittivity of 50, a loss tangent of 0.002 and density of $3.65 \mathrm{~g} / \mathrm{cm}^{3}$ over the frequency range $(200-2450 \mathrm{MHz})$. Microwave power is launched into the waveguide to excite it in its dominant $T E_{10}$-mode using a standard microwave technique [3]. Analyses have been carried out for three cases: 1) Single ORWDA, 2) a linear array of ORWDA having 3 number of elements, and 3) a planar 
array of ORWDA having $3 \times 3$ number of elements (Fig. 1).

According to the Kirchhoff-Huygens principle [4], the field, $E$, at an observing point, $P$, in a medium outside a surface, $A$, that encloses all the sources of the field, may be expressed in terms of the field, $\left(E_{s}, H_{\mathrm{s}}\right)$, on the surface, $\mathrm{A}$. The field over the aperture of an ORWDA is essentially linearly polarised: only a small fraction of the energy may be in the cross-polarisation. If this cross-polarisation component of the field is neglected, the problem may be reduced to that of scalar diffraction, with the assumption that the aperture dimensions are much longer than the wavelength in the muscle, $\lambda_{m}$. The analysis will be restricted to a rectangular plane aperture, where the aperture is taken in $x, y$ plane, and the electric field polarised in $y$-direction.

$E_{y}(p)=\frac{1}{4 \pi} \int_{-\frac{a}{2}}^{\frac{a}{2}} \int_{-\frac{b}{2}}^{\frac{b}{2}} E_{y} \psi\left[\left(\jmath \gamma_{m}^{*}+\frac{1}{r}\right) \hat{\imath}_{z} \cdot r+\jmath \gamma_{m}^{*} \hat{\imath}_{z} \cdot s\right] d x d y$

where, $\psi=e^{-\frac{j \gamma_{m}^{*} r}{r}}=$ the Green's function,

$r=\sqrt{\left(x-x_{0}\right)^{2}+\left(y-y_{o}\right)^{2}+\left(z-z_{o}\right)^{2}}=$ the distance between a source point in the aperture and the point, $\mathrm{P}$,

$\gamma_{m}^{*}=$ the complex propagation constant of the muscle,

$\hat{n}=$ the unit vector normal to $A$ ( $z$-direction).

$E_{y}=$ the aperture distribution,

$\hat{i_{z}}=$ the unit vector in the $z$-direction.

In order to compute the field for $z>0$ half space, the field at $z=0$ plane is assumed and modelled as half wavelength cosine function. The peak value is located at the center of ORWDA.

\section{Specific Absorption Rate (SAR) Distribution}

The specific absorption rate $(S A R)$, in the muscle under electromagnetic radiation is computed from the definition,

$S A R=\frac{\left|E_{y}(p)\right|^{2} \sigma_{m}}{2 \rho_{m}} \mathrm{~W} / \mathrm{kg}$

where, $\rho_{m} \approx 10^{3} \mathrm{Kg} / \mathrm{m}^{3}$, is the mass density of the tissue,

$\sigma_{m}$ is the conductivity of the muscle.

The $S A R$ distribution in the muscle are given for 1-watt power output from the aperture of 1,3 , and $3 \times 3$ elements ORWDAs along $x, y$ plane for a depth, $z=6 \mathrm{~cm}$, in the muscle. When the single ORWDA is in direct contact with the muscle, a hot spot near the aperture center, will be developed. This can hardly be overcomed by cooling the 
surface either from the ORWDA itself or by introducing a cooled bolus between the ORWDA and the muscle. The reduction in the effect of hot spot and better focusing of power may be achieved by using arrays of ORWDAs (Fig. 2).

The array specific absorption rate gain factor $G_{S A R}$ defined in [1], is considered as a design parameter,

$G_{S A R}=\frac{S A R_{\mathrm{a}}}{S A R_{s}}$

where the subscripts $a$ and $\mathrm{s}$ denotes the array and single applicator respectively. Arrays of ORWDAs can be seen to have a marked advantage over the single ORWDA. Significant levels of absorbed power are produced over a larger area beneath the applicator as shown in Fig. 3 .

\section{Conclusion}

It is found that single ORWDA may produce SAR distribution of wider and weaker field size at a depth, $z=6 \mathrm{~cm}$. The proposed arrays of ORWDAs have better focused and deeper SAR distribution capability than the single ORWDA. This in turn, implies that, less input power is required and hence there would be less power absorption in healthy tissues and in regions near the surface. Although, the best focusing effect may be achieved by using $3 \times 3$ elements ORWDA, that does not mean it is the optimal applicator because it requires bigger size. Both the size and complexity of geometry, increase with the depth of the tumour inside the muscle. Moreover, powering a number of ORWDAs is a problem relating to the design cost. In the array applicators under investigation, only a single oscillator and one measurement network are required. The array models currently adopted are simple and cheap compared to the phased array applicators which are widely used for deep hyperthermia.

\section{References}

[1] J. W. Hand, J. L. Cheetham and A. J. Hind, "Absorbed power distributions from coherent microwave arrays for localized hyperthermia, "IEEE Transactions on Microwave Theory and Techniques, vol. MTT-34, no. 5, pp. 484-489, 1986.

[2] C. Becerra and J. Rebollar, "Electric field distribution of waveguide arrays for local tumour hyperthermia," Journal of Microwave Power and Electromagnetic Encrgy, vol. 23, no. 4, pp. 247-254, 1988.

[3] R. E. Collin, Field Theory of Guided Waves, Mc. Graw-hill, New York, 1960.

[4] S. Silver, Microwave Antenna Theory and Design, Mc. Graw-hill, New York, 1949. 


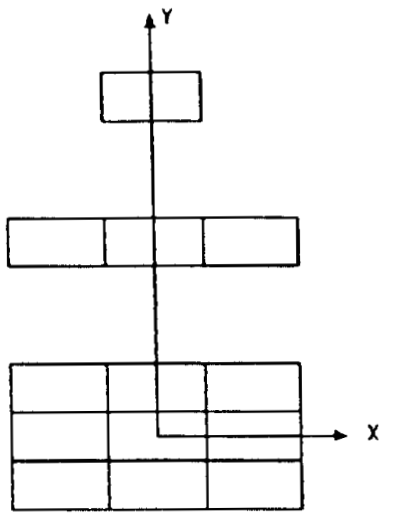

Fig. 1. Cross sections of three cases.

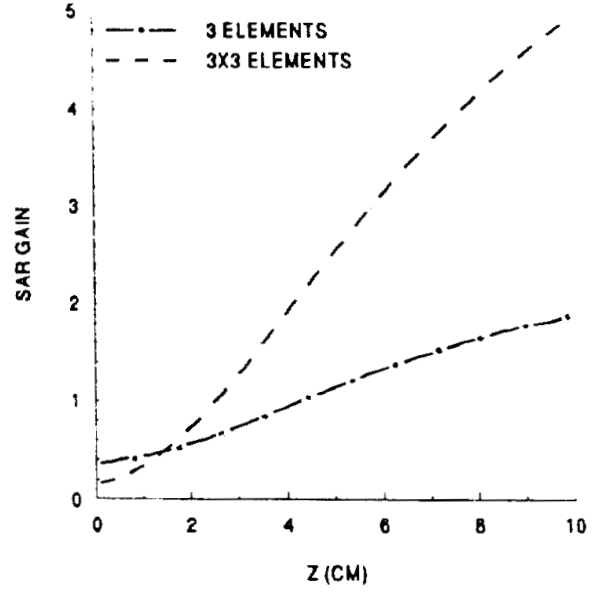

Fig. 3. SAR array gain factor along $z$-axis.
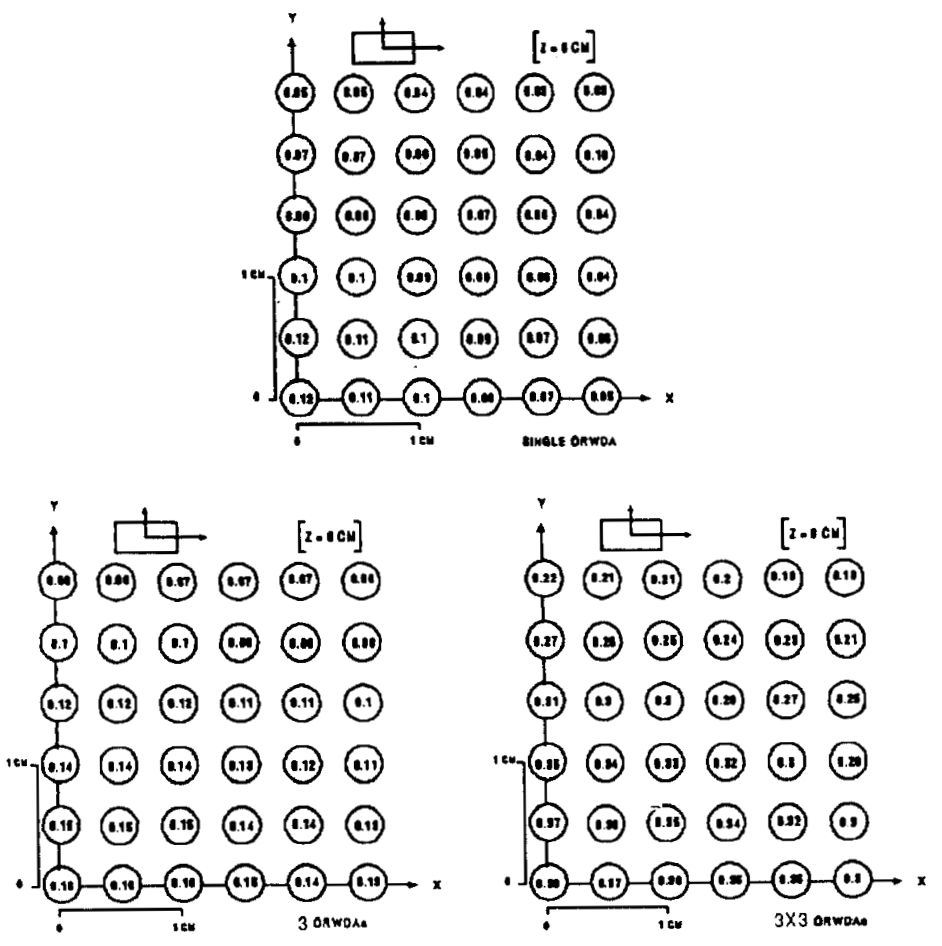

Fig. 2. SAR distributions along the $x, y$ axis. 